\title{
Teaching energy and sustainability: The Erice schools
}

\author{
erice-energy-school-2016.ct.infn.it
}

$\mathrm{Si}$ ince 2010, the Ettore Majorana Foundation and Centre for Scientific Culture (Erice, Italy) has been holding a biannual school on renewable energy and sustainability co-organized by the Materials Research Society (MRS) and E-MRS. This year, the European Physical Society, the Italian Physical Society, and the Eurosunmed Project joined the organization of the school, adding valuable contributions to this event.

The Ettore Majorana Foundation has sponsored world-class schools for more than 50 years. This year's International School of Solid State Physics was held from July 13 to 19 and was the union of the fifth course of "Materials for Energy and Sustainability" (MRS and E-MRS) and the third course of the "EPS-SIF International School on Energy." The aim of the school is to present the state of the art and future perspectives for materials applied to the generation and storage of renewable and sustainable energy. Among the directors of this school (David Cahen, Luisa Cifarelli, David Ginley, Abdelilah Slaoui, Antonio Terrasi, and Friedrich Wagner) are presidents and board members of these learned societies.

Seventy-five doctoral students and young researchers from 14 different countries experienced seven days of "full immersion" in the field of energy and sustainability. They attended lectures covering some of the most important and strategic topics in the field of generation, storage, and consumption of energy, which aimed at putting these topics in perspective in terms of sustainability, with an emphasis on life cycles. In addition, the students were given projects, which they worked on in small groups and reported on at the end of the school.

Thanks to distinguished speakers from France, Germany, Israel, Italy, The Netherlands, Switzerland, and the
United States, the school gave a wide overview of energy challenges, including technologies, critical materials, pollution, safety, and economy. Teaching was accomplished through lectures and close interactions with students during the breaks, lunches, dinners, and poster sessions. Lectures focused on global climate, $\mathrm{CO}_{2}$ sequestration, water-energy nexus, nuclear energy, solar power, both thermal and photovoltaic, as well as photosynthesis and biofuels, geothermal and wind energy, various forms of energy storage, thermoelectricity, the future of the electrical grid, and how to view mobility and buildings from the point of view of energy and sustainability. Students were continuously stimulated to interact and to solve problems and exercises.

Their main project was to gather in teams of five to seven people and critically assess papers from MRS Energy \& Sustainability-A Review Journal, which publishes reviews on key energy and sustainability topics in materials research and development. At the end of the school, each team presented its analysis of "their" paper, having done fact- and logic-checking (i.e., serving as critical readers). An important aim of the school was to provide the students with the background needed to have a critical, not passive, attitude when involved in their field of research while using their familiarity with the scientific method.

The school is a great opportunity for students and postdoctoral fellows from around the world to meet with and learn from their peers and established experts in their area of study.

Antonio Terrasi University of Catania, Italy

Group photo of participants taken in the Dirac Hall of the Ettore Majorana Centre. 